\title{
AVALIAÇÃO ESPAÇO-TEMPORAL DAS ARMAZENAGENS GRAVIMÉTRICA E VOLUMÉTRICA DA ÁGUA NUM LATOSSOLO COM CITROS ${ }^{(1)}$
}

\author{
Dolorice Moreti $^{(2)}$, Paulo Leonel Libardi ${ }^{(3)}$, Genelício Crusoé \\ Rocha $^{(4)}$, Márcio José Lovatti ${ }^{(5)}$ \& Laura Ianello Giassetti Aguiar ${ }^{(6)}$
}

\begin{abstract}
RESUMO
A estabilidade temporal é descrita como a persistência temporal de um padrão espacial e é avaliada pela análise de correlação de medidas em datas sucessivas. Neste trabalho, foi avaliada a armazenagem de água no solo em duas transeções de $76 \mathrm{~m}$ cada, contendo 20 pontos em cada uma. em um Latossolo Vermelho-Amarelo argissólico cultivado com citros com 11 anos em Piracicaba/SP. A umidade do solo para determinação da armazenagem, na camada de 0,00-1,10 m, ao longo do tempo, foi obtida com uma sonda de nêutrons, calibrada para o solo em estudo, realizando leituras semanais às profundidades de $0,20,0,40,0,60,0,80,1,0$ e $1,10 \mathrm{~m}$. A armazenagem de água no solo foi determinada utilizando-se o método de Simpson. Os dados da armazenagem média de água no solo nos três anos de estudo mostraram que sua distribuição, ao longo do tempo, apresentou comportamento semelhante e que os coeficientes de correlação linear e de posição de Spearman foram altos entre o ano 1 e o ano 2, o ano 2 e o ano 3 e o ano 1 e o ano 3, indicando existência de estabilidade temporal e persistência temporal significativa do padrão espacial observado. Foi verificada dependência espacial moderada de $17,10 \mathrm{~m}$ pelo semivariograma exponencial. Pela técnica da diferença relativa, os pontos que apresentaram comportamento estável no tempo foram o ponto 29 , para os anos $1 \mathrm{e}$ 2, e os pontos 16, 29 e 39, para o ano 3, que podem ser usados para estimar com
\end{abstract}

\footnotetext{
(1) Parte da Tese de Doutorado do Primeiro autor. Recebido para publicação em julho de 2006 e aprovado em julho de 2007.

${ }^{(2)}$ Pós-Graduando do Departamento de Solos e Nutrição de Plantas, Escola Superior de Agricultura "Luiz de Queiroz" - ESALQ/ USP. Av. Pádua Dias 11, Caixa Postal 9, CEP 13428-900 Piracicaba (SP). E-mail:dmoreti@esalq.usp.br

${ }^{(3)}$ Professor Titular do Departamento de Ciências Exatas, ESALQ/USP. E-mail:pllibardi@esalq.usp.br

(4) Professor Titular do Centro de Ensino Superior dos Campos Gerais - CESCAGE. Av Carlos Cavalcanti 8000, Bairro Uvaranas, CEP 84900-000 Ponta Grossa (PR). E-mail: genelicio@cescage.com.br

(5) Estudante de Graduação do Curso de Engenharia Florestal, ESALQ/USP. E-mail: mjlovatti@bol.com.br

(6) Pós-Graduando do Programa de Agricultura Tropical e Subtropical, área de concentração de Recursos Agroambientais do Instituto Agronômico. Caixa Postal 28, CEP 13020902 Campinas (SP). E-mail: lauragiassetti@gmail.com
} 


\begin{abstract}
segurança a armazenagem média de água no solo. $O$ ponto 29 foi o que se apresentou estável para os três anos de estudo, sendo, portanto, o mais recomendável tanto para a armazenagem gravimétrica como para a volumétrica.
\end{abstract}

Termos de indexação: variabilidade, transeção, sonda de nêutrons.

\title{
SUMMARY: SPATIAL AND TEMPORAL EVALUATION OF GRAVIMETRIC AND VOLUMETRIC WATER STORAGE IN AN OXISOL UNDER CITRUS
}

\begin{abstract}
Temporal stability is described as the temporal persistence of a spatial pattern and is evaluated through correlation analysis of successive measurement data. In this study, the soil water storage was evaluated along two $76 \mathrm{~m}$ transects, each of them sampled at 20 points. The soil is an Oxisol (Yellow Red Latosol) under an 11-year-old citrus orchard, in Piracicaba-SP, Brazil. Soil water content, for the calculation of soil water storage in the layer $0.0-1.10 \mathrm{~m}$, was obtained from weekly neutron probe readings at depths of $0.20,0.40$, $0.60,0.80,1.0$ and $1.10 \mathrm{~m}$. Soil water storage was calculated by Simpson's method. The mean soil water storage for the three study years showed similar distribution over time. The linear correlation coefficient and Spearman rank correlation were high between year 1 and 2, year 2 and 3, and year 1 and 3, indicating temporal stability and a significant temporal persistence of the spatial pattern observed. A moderate space dependence of $17.10 \mathrm{~m}$ was verified for the exponential semivariogram. For the relative difference technique the following points presented stable behavior over time: point 29 for years 1 and 2, and points 16, 29 and 39 for year 3. These points can be used to estimate the mean soil water storage. Point 29 is the most recommendable for both gravimetric and volumetric soil water storage because of the stability in the three studied years.
\end{abstract}

Index terms: variability, transect, neutron probe.

\section{INTRODUÇÃO}

A quantidade de água consumida por uma cultura agrícola, durante o seu desenvolvimento, varia com a distribuição espacial e temporal do clima, da variedade da cultura e do manejo do sistema agrícola, que altera as propriedades físicas do solo diretamente relacionadas à armazenagem de água. É importante o conhecimento de como as plantas utilizam a água retida no solo e de como respondem à armazenagem, principalmente em estudos da agricultura de precisão, com base em princípios de gerenciamento agrícola de informação sobre as variabilidades espacial e temporal dos fatores de produção e da produtividade (Mercante et al., 2003).

A variação da armazenagem de água na planta é conseqüência da defasagem temporal entre a absorção de água e a perda na fase de vapor pela planta, que conduz ao desenvolvimento de déficits hídricos a curto e a longo prazo, os quais dependem de um conjunto de fatores que afetam a absorção e transpiração (Angelocci, 2002). Portanto, é importante o conhecimento do solo no que diz respeito às propriedades que influenciam a retenção de água.

A variabilidade espacial da armazenagem de água no solo está associada a uma série de propriedades, que variam no espaço - profundidade do solo, capacidade de infiltração de água no solo, topografia, fertilidade, ocorrência de pragas e doenças e microclima (Gonçalves et al., 2001).

A condutividade hidráulica apresenta grande variabilidade espacial e está diretamente relacionada com a drenagem interna no solo. Ruiz \& Utset (1999) verificaram coeficiente de variação de $120,5 \%$ e Warrick \& Nielsen (1980), de $420 \%$. Mallants et al. (1996), estudando a variabilidade espacial das propriedades hidráulicas do solo, obtiveram coeficiente máximo de variação para a condutividade hidráulica saturada de 599,322 e $879 \%$ para as profundidades de $0,1,0,5$ e $0,9 \mathrm{~m}$, rescpectivamente; para o conteúdo residual de água e porosidade no solo a variação foi de 53 e $156 \%$, respectivamente, necessitando, com isso, de grande número de amostras para bem representar a área do local de estudo, o que se reflete em alto custo e tempo.

Em face de as análises da variabilidade espacial do solo no campo necessitarem de grande número de observações, pesquisas para o desenvolvimento de técnicas, a fim de minimizar o número de observações sem prejuízo das informações, tornaram-se prioritárias. Com isso, Vachaud et al. (1985) introduziram um método para reduzir o número de 
observações, de forma a caracterizar o comportamento do solo no campo, definindo o conceito de estabilidade temporal como a associação invariável no tempo entre local no espaço e valores de parâmetros da estatística clássica. Seus dados mostraram que a variabilidade espacial do conteúdo de água no solo no campo pode ser explicada pela variabilidade da textura do solo: os locais com maior teor de argila permaneceram sempre mais úmidos ao longo do tempo.

Kachanoski \& De Jong (1988), trabalhando na mesma linha, aprofundaram o estudo da dependência de escala da estabilidade temporal para avaliar as alterações espacial e temporal da armazenagem de água no solo. A estabilidade temporal foi avaliada pela análise de correlação de medidas em datas sucessivas, e a análise de coerência foi sugerida como um método para examinar a persistência temporal. Os resultados mostraram que o padrão espacial da armazenagem de água no solo em pequenas escalas foi alterado pela recarga, mas na secagem esse padrão não foi alterado.

O objetivo deste trabalho foi avaliar a variabilidade espaço-temporal da armazenagem de água em um Latossolo Vermelho-Amarelo cultivado com citros.

\section{MATERIAL E MÉTODOS}

O estudo experimental foi realizado em uma área de citros do campus Luiz de Queiroz da Universidade de São Paulo, em Piracicaba-SP (22 ${ }^{\circ} 42^{\prime} 43$ ” S, $47^{\circ} 37^{\prime} 10^{\prime} \mathrm{W}$ e $546 \mathrm{~m}$ de altitude). O solo é um Latossolo Vermelho-Amarelo argissólico (Embrapa, 1999), relevo suave, com declividade máxima de $3 \%$. A parcela experimental constituiu-se de duas transeções (duas linhas) com 20 pontos de observação espaçados de $4,0 \mathrm{~m}$, cada um deles localizado no centro da distância entre duas plantas ao longo da linha. A cultura de citros foi implantada em março de 1991, num espaçamento de 4,0 m entre plantas e 7,0 m entre linhas, com o sentido do plantio em nível. Rocha et al. (2004) avaliaram a textura desse solo, nos quarenta pontos de observação, até a profundidade de $1,10 \mathrm{~m}$, com incrementos de $0,10 \mathrm{~m}$; os resultados entre pontos não apresentaram diferenças significativas, demonstrando uniformidade. A cultivar é a Valência (Citrus sinensis L. Osbeck), sobre o porta-enxerto Cleópatra. Em cada ponto de observação foi instalado um tubo de $\mathrm{Al}$ de $1,50 \mathrm{~m}$ de comprimento $(1,20 \mathrm{~m}$ abaixo da superfície do solo), com $45 \mathrm{~mm}$ de diâmetro interno e 1,5 $\mathrm{mm}$ de espessura de parede, para acesso de uma sonda de nêutrons, modelo Hydroprobe 503CNP Coorporation. Com a sonda de nêutrons foram realizadas leituras semanais às profundidades de 0,20 , $0,40,0,60,0,80,1,0$ e $1,10 \mathrm{~m}$, para cálculo da armazenagem de água no solo no perfil de $0,00-1,10 \mathrm{~m}$ ao longo do tempo, após sua calibração $(r=0,87)$. A armazenagem volumétrica de água, ou simplesmente armazenagem de água no solo na camada de $0-1,10 \mathrm{~m}$ $\left(\mathrm{h}_{1,10}\right)$, foi determinada utilizando-se os dados de conteúdo de água, obtidos a partir das leituras realizadas com a sonda de nêutrons, pelo método de Simpson (Libardi, 2005), segundo a equação:

$$
\begin{gathered}
h_{1,10}=\left(0,2 . \theta_{0,20}\right)+0,20 /{ }_{3}\left[\theta_{0,20}+\left(4 \cdot \theta_{0,40}\right)+\right. \\
\left.\left(2 . \theta_{0,60}\right)+\left(4 . \theta_{0,80}\right)+\theta_{1,0}\right]+\left(0,1 . \theta_{1,0}\right)
\end{gathered}
$$

em que $\mathrm{h}_{1,10}$ é expresso em $\mathrm{m}$ de água e $\theta$ é a umidade volumétrica, com seu subíndice sendo a profundidade (m) de medida.

O presente trabalho foi desenvolvido por um período de três anos. Durante os dois primeiros, Rocha et al. (2005) desenvolveram estudos na mesma área, mas calculando a armazenagem de água a partir de medidas de umidade à base de massa. Nesse trabalho, em cada um dos 40 pontos da transeção estudada, foram retiradas amostras com estrutura indeformada ao longo do perfil de solo, para determinação da densidade do solo, com o que se pôde refazer os cálculos (para os dois primeiros anos) e apresentar os dados novos para o terceiro ano, calculando a armazenagem a partir de valores de umidade à base de volume.

O período de avaliação foi de três anos: 2001/2002, 2002/2003 e 2003/2004, denominados ano 1, ano 2 e ano 3 , respectivamente. Das respectivas profundidades foram coletadas amostras indeformadas, para determinação da densidade do solo (Embrapa, 1997) utilizada no cálculo da umidade e armazenagem volumétricas.

Para análise, os dados foram primeiramente interpretados com base na análise descritiva (Libardi et al., 1996), utilizando-se o software Statistica (STATSOFT, 2005). Observado o comportamento geral dos dados por esta análise, procedeu-se, então, à sua avaliação e interpretação, conforme a metodologia geoestatística (Matheron, 1971), obtendo-se o semivariograma experimental; foi utilizado o programa GeoR (Ribeiro Júnior \& Diggle, 2001) para verificação da dependência espacial da armazenagem de água no solo.

$\mathrm{Na}$ avaliação da estabilidade temporal da armazenagem de água no solo foram utilizados dois índices: o coeficiente de posição de Spearman e a diferença relativa (Vachaud et al., 1985). Utilizou-se também o coeficiente de correlação (r), a inclinação e o intercepto das regressões lineares entre medidas em tempos sucessivos (Kachanoski \& De Jong, 1988).

O coeficiente de Spearman, $r_{s}$, é dado por:

$$
r_{s}=1-\frac{6 \sum_{i=1}^{n}\left(R_{i j}-R_{i j}\right)}{n\left(n^{2}-1\right)}
$$

em que n é o número de amostragens (locais ou pontos de observação); $R_{\mathrm{ij}}$, a posição da variável $\mathrm{S}_{\mathrm{ij}}$ observada no local i na data j; $\mathrm{e} \mathrm{R}_{\mathrm{ij}}$, a posição da mesma variável, no mesmo local, mas na data ${ }_{j}$. Um valor $r_{s}=1$ 
corresponde a uma perfeita estabilidade entre os tempos j e j', e quanto mais próximo for o $r_{\mathrm{s}}$ de 1 , maior a estabilidade do processo.

A diferença relativa $\left(\delta_{i j}\right)$ corresponde à diferença $\Delta_{i j}$ entre a determinação individual da armazenagem de água $\left(\mathrm{S}_{i j}\right)$ no local $i$ e no tempo $j$ e a média da armazenagem de água $\left(\bar{S}_{j}\right)$ no mesmo tempo, dividida por $\bar{S}_{j}$, isto é:

$$
\delta_{i j}=\frac{\Delta_{i j}}{\bar{S}_{j}}
$$

sendo:

$$
\Delta_{i j}=S_{i j}-\bar{S}_{j}
$$

$\mathrm{e}$

$$
\bar{S}_{j}=\left(\frac{1}{n}\right) \sum_{i=1}^{i=n} S_{i j}
$$

em que n é o número de pontos (locais) de amostragem.

Quando essa diferença relativa apresentar pequena variação temporal entre as posições, é indicação de estabilidade temporal. A média no tempo dessa diferença relativa para cada local $i$, ou diferença relativa média, associada ao respectivo desvio-padrão, permite identificar uma ou mais posições que representam a média geral do campo, assim como os valores sub e superestimados: quando o desvio-padrão for menor, maior é a confiabilidade de utilizar o referido ponto para estimar a média geral.

\section{RESULTADOS E DISCUSSÃO}

À semelhança do que se encontrou para a armazenagem gravimétrica nos dois primeiros anos (Rocha et al., 2005), verifica-se, para a volumétrica, nos três anos, que os valores das medidas de tendência central (média e mediana) foram semelhantes, indicando simetria na distribuição dos dados em todos os pontos analisados (Quadro 1). Os valores de assimetria e curtose também ficaram próximos a zero, indicando que os dados se aproximam da normal, sendo aceitos os valores que ficam entre $-2 \mathrm{e}+2$. Os coeficientes de curtose - quase todos negativos e com valor máximo absoluto próximo de 1 - levam a classificar as distribuições apresentadas no quadro 1 como leptocúrticas; em Rocha et al. (2005) elas foram caracterizadas como platicúrticas. Os coeficientes de variação para os três anos de observação ficaram entre 10,64 e 15,66 \%; segundo Andriotti (2004), valores de coeficientes de variação abaixo de $40 \%$ refletem homogeneidade da amostra.

Uma vez que a estatística descritiva mostrou normalidade dos dados e a estacionaridade não pôde ser testada estatisticamente, tendências de concentração de valores ou de variação em determinada direção não podem ser identificadas, embora a umidade varie no espaço (Gonçalves et al., 1999). Portanto, com base nessas observações, admite-se que a estacionaridade descrita pela hipótese intrínseca seja aceitável. Assim, foi verificada a dependência espacial para a armazenagem de água durante os três anos de observação, utilizando o programa GeoR versão 1.5-5 (Ribeiro Júnior \& Diggle, 2001). O ajuste dos semivariogramas permitiu verificar qual o alcance do parâmetro em estudo, ou seja, que distância poderia ser utilizada para realização das amostragens sem prejudicar os resultados, sendo essa distância a dependência amostral. O semivariograma foi ajustado pela verossimilhança dos dados, usando a função de correlação exponencial, e suavizado, condicionado ao atendimento da condição de estacionaridade dos dados caracterizada como hipótese intrínseca.

A dependência espacial foi de 16,92; 16,20; 18,11; e $17,10 \mathrm{~m}$ para o ano 1 , ano 2 , ano 3 e a média dos três anos, respectivamente. Na análise do grau de dependência espacial das variáveis foi utilizada a classificação de Cambardella et al. (1994), em que são considerados de forte dependência espacial os semivariogramas que têm efeito pepita $\leq 25 \%$ do patamar; moderada, quando entre 25 e $75 \%$; e fraca, quando $>75 \%$. Para os referidos dados, a dependência espacial foi classificada como moderada, visto que a relação efeito pepita e patamar é de 48, 43 e $57 \%$ para o ano 1, ano 2 e ano 3, respectivamente (Quadro 2).

$\mathrm{O}$ alcance variou de 16 a $18 \mathrm{~m}$ e o efeito pepita correspondeu de 43 a $57 \%$ do patamar, sendo, portanto, a estrutura de dependência espacial responsável por cerca de 57 a $43 \%$ da variação total.

Quanto ao comportamento da armazenagem média de água no solo para os três anos, verifica-se que, apesar de os valores em cada ponto não serem os mesmos, a sua distribuição ao longo do tempo, tanto para o período de recarga como para secagem, apresenta comportamento semelhante, indicando a existência de estabilidade temporal (Figura 2). Gonçalves et al. (1999) encontraram valores de umidade com distribuição espacial estável no tempo, durante o período de secagem, na faixa que usualmente ocorre em solos irrigados. Nesse sentido, Rocha et al. (2005) também encontraram valores de armazenagem da água com padrão espacial estável ao longo do tempo, tanto no período de recarga quanto no de secagem do solo.

Os coeficientes de correlação linear e de posição foram altos entre o ano 1 e o ano 2 , ano 1 e o ano 3 e o ano 2 e o ano 3 (Quadro 3), indicando a existência de estabilidade temporal e uma persistência temporal significativa do padrão espacial observado. Os coeficientes de correlação de Spearman foram altos para os três anos, correspondendo a uma perfeita estabilidade $(0,1 \%)$ entre os tempos de estudo, pois, quanto mais próximo de 1 o seu valor, maior é a estabilidade. 
Quadro 1. Resumo estatístico dos valores de armazenagem da água no solo (m) em cada ponto, para o período de amostragem

Ponto Média Mediana L. máx. L. mín. Amp.total Desvio-padrão Curtose Assimetria CV

\begin{tabular}{|c|c|c|c|c|c|c|c|c|c|}
\hline & & & & & & & & & $\%$ \\
\hline 1 & 0,18 & 0,18 & 0,27 & 0,13 & 0,14 & 0,028 & 0,08 & $-0,54$ & 15,66 \\
\hline 2 & 0,17 & 0,17 & 0,22 & 0,13 & 0,09 & 0,023 & $-1,01$ & $-0,45$ & 13,44 \\
\hline 3 & 0,18 & 0,18 & 0,23 & 0,14 & 0,09 & 0,024 & $-0,82$ & $-0,44$ & 13,04 \\
\hline 4 & 0,18 & 0,19 & 0,23 & 0,14 & 0,09 & 0,023 & $-0,90$ & $-0,38$ & 12,33 \\
\hline 5 & 0,19 & 0,19 & 0,24 & 0,14 & 0,10 & 0,024 & $-0,43$ & $-0,41$ & 12,59 \\
\hline 6 & 0,17 & 0,18 & 0,22 & 0,13 & 0,09 & 0,022 & $-0,86$ & $-0,43$ & 12,60 \\
\hline 7 & 0,17 & 0,17 & 0,21 & 0,13 & 0,08 & 0,020 & $-0,68$ & $-0,24$ & 11,70 \\
\hline 8 & 0,16 & 0,16 & 0,21 & 0,13 & 0,08 & 0,021 & $-0,98$ & $-0,18$ & 12,93 \\
\hline 9 & 0,17 & 0,17 & 0,22 & 0,13 & 0,09 & 0,021 & $-0,46$ & $-0,29$ & 12,13 \\
\hline 10 & 0,18 & 0,18 & 0,22 & 0,14 & 0,08 & 0,019 & $-0,43$ & $-0,25$ & 10,64 \\
\hline 11 & 0,16 & 0,16 & 0,21 & 0,13 & 0,08 & 0,020 & $-0,95$ & $-0,09$ & 12,53 \\
\hline 12 & 0,17 & 0,17 & 0,22 & 0,13 & 0,09 & 0,021 & $-0,77$ & $-0,38$ & 12,80 \\
\hline 13 & 0,17 & 0,17 & 0,22 & 0,13 & 0,09 & 0,022 & $-0,76$ & $-0,33$ & 12,67 \\
\hline 14 & 0,17 & 0,17 & 0,22 & 0,14 & 0,09 & 0,022 & $-0,91$ & $-0,30$ & 12,83 \\
\hline 15 & 0,15 & 0,15 & 0,20 & 0,12 & 0,07 & 0,018 & $-0,74$ & $-0,01$ & 12,05 \\
\hline 16 & 0,17 & 0,17 & 0,22 & 0,14 & 0,08 & 0,020 & $-0,63$ & $-0,14$ & 11,35 \\
\hline 17 & 0,16 & 0,16 & 0,20 & 0,13 & 0,08 & 0,019 & $-0,81$ & $-0,02$ & 12,07 \\
\hline 18 & 0,16 & 0,16 & 0,20 & 0,13 & 0,08 & 0,020 & $-0,97$ & 0,11 & 12,22 \\
\hline 19 & 0,17 & 0,18 & 0,22 & 0,13 & 0,08 & 0,021 & $-0,73$ & $-0,16$ & 12,16 \\
\hline 20 & 0,17 & 0,17 & 0,21 & 0,13 & 0,08 & 0,020 & $-0,64$ & $-0,12$ & 11,80 \\
\hline 21 & 0,18 & 0,18 & 0,22 & 0,14 & 0,09 & 0,024 & $-1,09$ & $-0,39$ & 13,41 \\
\hline 22 & 0,17 & 0,17 & 0,26 & 0,13 & 0,13 & 0,025 & 0,35 & $-0,09$ & 14,76 \\
\hline 23 & 0,17 & 0,17 & 0,22 & 0,13 & 0,09 & 0,024 & $-1,10$ & $-0,09$ & 14,24 \\
\hline 24 & 0,18 & 0,18 & 0,22 & 0,13 & 0,08 & 0,022 & $-0,89$ & $-0,55$ & 12,63 \\
\hline 25 & 0,18 & 0,18 & 0,22 & 0,13 & 0,09 & 0,024 & $-0,89$ & $-0,53$ & 13,37 \\
\hline 26 & 0,17 & 0,17 & 0,21 & 0,13 & 0,08 & 0,021 & $-1,01$ & $-0,38$ & 12,57 \\
\hline 27 & 0,16 & 0,16 & 0,21 & 0,13 & 0,09 & 0,022 & $-0,98$ & $-0,06$ & 13,24 \\
\hline 28 & 0,17 & 0,17 & 0,22 & 0,13 & 0,09 & 0,022 & $-0,82$ & $-0,48$ & 13,15 \\
\hline 29 & 0,17 & 0,18 & 0,21 & 0,13 & 0,08 & 0,021 & $-0,63$ & $-0,78$ & 12,43 \\
\hline 30 & 0,17 & 0,17 & 0,21 & 0,13 & 0,08 & 0,021 & $-0,76$ & $-0,54$ & 12,40 \\
\hline 31 & 0,17 & 0,18 & 0,21 & 0,14 & 0,08 & 0,022 & $-1,08$ & $-0,61$ & 12,45 \\
\hline 32 & 0,18 & 0,18 & 0,22 & 0,14 & 0,08 & 0,021 & $-0,70$ & $-0,51$ & 11,76 \\
\hline 33 & 0,17 & 0,17 & 0,21 & 0,11 & 0,10 & 0,021 & $-0,34$ & $-0,33$ & 12,10 \\
\hline 34 & 0,17 & 0,17 & 0,21 & 0,13 & 0,08 & 0,019 & $-0,85$ & $-0,01$ & 11,27 \\
\hline 35 & 0,17 & 0,17 & 0,21 & 0,13 & 0,08 & 0,020 & $-0,82$ & $-0,51$ & 12,17 \\
\hline 36 & 0,17 & 0,17 & 0,21 & 0,13 & 0,08 & 0,021 & $-0,87$ & $-0,59$ & 12,36 \\
\hline 37 & 0,16 & 0,17 & 0,21 & 0,12 & 0,09 & 0,023 & $-0,95$ & $-0,37$ & 14,10 \\
\hline 38 & 0,17 & 0,18 & 0,22 & 0,13 & 0,09 & 0,021 & $-0,79$ & $-0,73$ & 12,17 \\
\hline 39 & 0,17 & 0,17 & 0,21 & 0,13 & 0,09 & 0,021 & $-0,72$ & $-0,42$ & 12,39 \\
\hline 40 & 0,17 & 0,17 & 0,21 & 0,13 & 0,07 & 0,018 & $-0,64$ & $-0,37$ & 10,84 \\
\hline
\end{tabular}

Quadro 2. Coeficientes do modelo ajustado aos semivariogramas para os anos de observação

\begin{tabular}{|c|c|c|c|c|c|c|}
\hline Ano & Alcance & Efeito pepita & Estrutura & Patamar & Efeito pepita/Patamar & Modelo \\
\hline & $\underline{\ldots}$ & 2 & 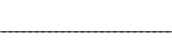 & - & $\%$ & \\
\hline 1 & 17 & 0.24 & 0.26 & 0.50 & 48 & exponencial \\
\hline 2 & 16 & 0.14 & 0.18 & 0.32 & 43 & exponencial \\
\hline 3 & 18 & 0.36 & 0.27 & 0.64 & 57 & exponencial \\
\hline 3 anos & 17 & 0.25 & 0.19 & 0.44 & 57 & exponencial \\
\hline
\end{tabular}



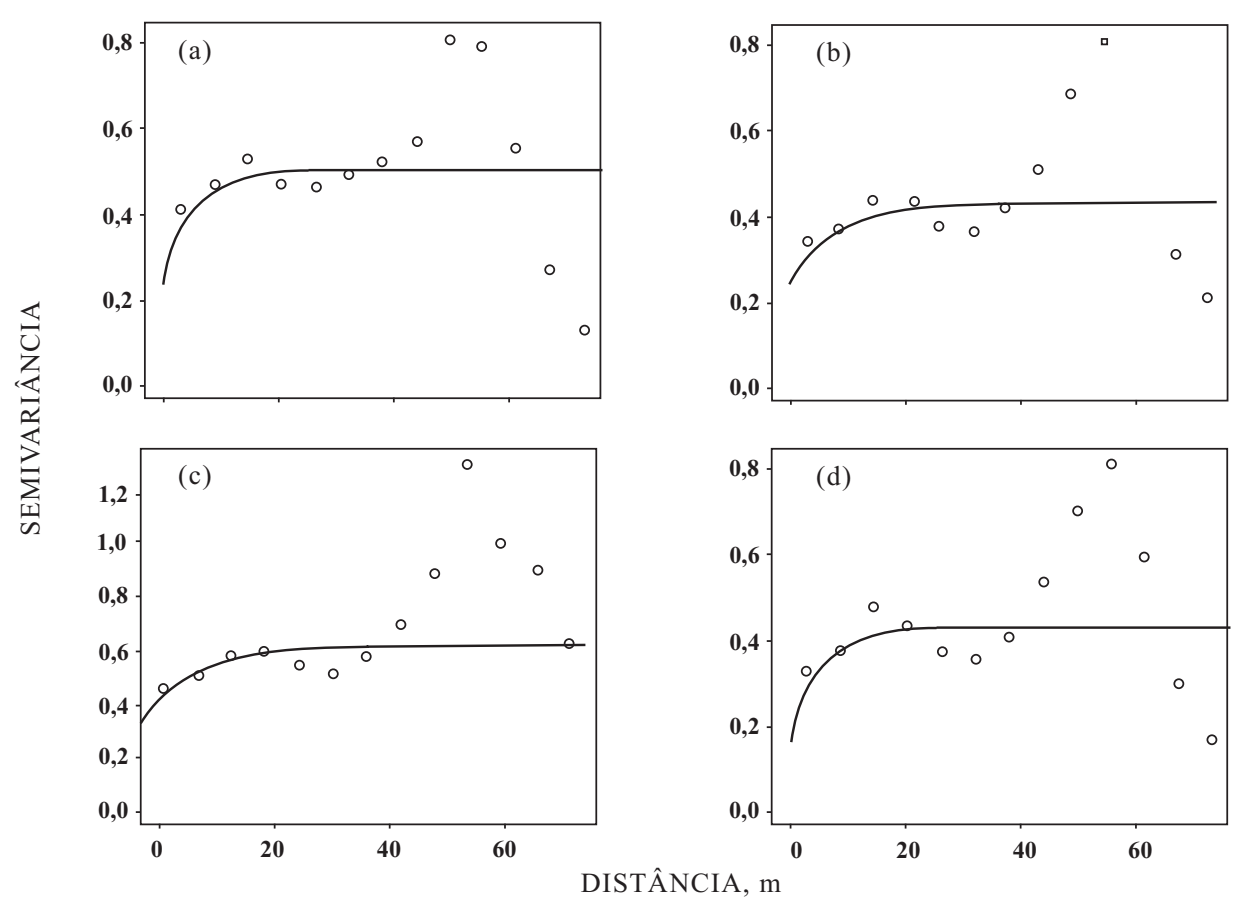

Figura 1. Semivariogramas para armazenagem média no tempo do ano 1 (a), ano 2 (b) ano 3 (c) e média dos três anos (d), para o armazenamento de água no solo.

O coeficiente de determinação $\left(R^{2}\right)$ indica o quanto da variância espacial observada no tempo $t_{2}$ pode ser

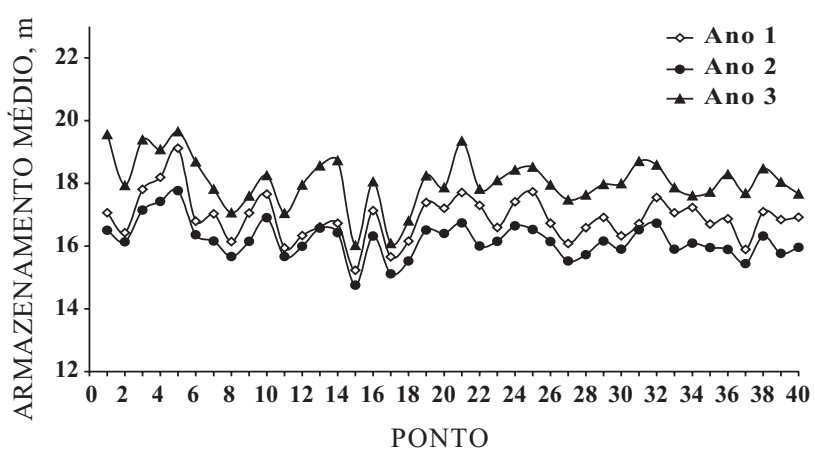

Figura 2. Armazenagem média de água no solo durante os três anos de observação. explicada pela variância no tempo $t_{1}$, isto é, a persistência do padrão espacial. Assim, verifica-se que $83 \%$ da variação no ano 2 é explicada pela variância do ano 1 e $77 \%$ da variabilidade espacial do ano 3 é explicada pela variabilidade do ano 2 . Conclusões semelhantes podem ser obtidas com relação às posições das observações de armazenagem a partir dos coeficientes de posição. Apesar da da persistência temporal significativa do padrão espacial e de as posições terem permanecidos quase as mesmas, a diferença relativa $\delta(j)$ não permaneceu constante. A inclinação e o intercepto da regressão (Quadro 4) são diferentes $(p<0,05)$ dos valores esperados se a diferença relativa $\delta(j)$ fosse constante (Kaschanoski $\&$ De Jong, 1988).

A inclinação e o intercepto deveriam ser de 0,94 e 0 e 1,125 e 0 entre os anos 1 e 2 e 2 e 3 , em vez de 0,0387 , $0,73,0,016$ e 1,22 , respectivamente. Os coeficientes de

\section{Quadro 3. Parâmetros estatísticos para armazenagem de água no solo (0-1,10 m)}

\begin{tabular}{|c|c|c|c|c|c|c|}
\hline \multirow{2}{*}{ Período } & \multirow{2}{*}{ Média } & \multirow{2}{*}{ Desvio-padrão } & \multirow{2}{*}{$\mathrm{CV}$} & \multicolumn{3}{|c|}{ Coeficiente de correlação ${ }^{(1)}$} \\
\hline & & & & Ano 1 & Ano 2 & Ano 3 \\
\hline & $\mathrm{m}$ & & $\%$ & & & \\
\hline Ano 1 & 0,17 & 0,007 & 4,26 & 1 & $0,91(0,81)^{*}$ & $0,78(0,64)^{*}$ \\
\hline Ano 2 & 0,16 & 0,006 & 3,58 & & 1 & $0,88(0,85)^{*}$ \\
\hline Ano 3 & 0,18 & 0,008 & 4,43 & & & 1 \\
\hline
\end{tabular}

${ }^{(1)}$ Coeficiente de correlação de Spearman (valor crítico para $n=40$ e $0,1 \%$ de probabilidade é 0,49 ). 
correlação linear e de posição para as medidas de ano 1 e 2 e ano 2 e 3 são significativos para $\mathrm{p}<0,05$, mas $73 \%$ do padrão espacial persistiu durante o período analisado.

Com relação ao desvio relativo médio, conforme Vachaud et al. (1985), verifica-se que os desviospadrões foram muito baixos, variando de 0,010 a 0,029 para os três anos, mostrando a confiabilidade dos valores da diferença relativa. É importante lembrar que as diferenças relativas médias, associadas ao desvio-padrão no tempo, permitem identificar a posição cujos valores se aproximam da média em qualquer momento e que, quanto menor o desvio-padrão correspondente, maior a confiabilidade da medida naquele ponto para estimar a média geral.

O gráfico resultante da diferença relativa para as medidas ranqueadas (Figura 3) permite visualizar os valores da $\bar{\delta}_{i}$ (diferença relativa média) para a armazenagem de água no solo dos três anos; os valores de $\bar{\delta}_{i}$ estão listados do menor para o maior. Note que há valores que ou subestimam $\left(\bar{\delta}_{i}<0\right)$ ou superestimam $\bar{\delta}_{i}>0$ ) a armazenagem média de água no campo, independentemente da época da observação.

Verifica-se que o valor da armazenagem de água no local 15 é 9,90; 8,85; e 11,26 \% menor que a média do primeiro, segundo e terceiro anos, respectivamente, enquanto no local 5 é 13,$15 ; 9,74$; e $8,83 \%$ maior que a média para o primeiro, segundo e terceiro anos, respectivamente. Portanto, os pontos que mais se distanciaram da média, apresentando menor e maior armazenagem, foram os mesmos para os três anos. Os valores que estão mais próximos da média apresentaram variação de um ano para outro, mas alguns pontos tiveram o mesmo comportamento. Para o ano 1 e ano 2 , o ponto 29 foi o que apresentou valor mais próximo da média, com diferença relativa de 0,07 e 0,16\% e desvio-padrão de 0,021 e 0,025, respectivamente. Para o ano 3, foram os pontos 16 , com diferença relativa de $0,02 \%$ e desvio-padrão de 0,$017 ; 39$, com diferença relativa de $0,06 \%$ e desvio-padrão correspondente de 0,014; e também o 29, com diferença relativa de $0,48 \%$ e desvio-padrão de 0,014 , os mais próximos da média. Portanto, o ponto 29 poderia ser utilizado para monitoramento da umidade no solo. No trabalho de Rocha et al. (2005) foi identificado o ponto 28, para os anos 1 e 2 .

Esses dados referentes à diferença relativa média mostram que, no geral, o fato de utilizar os valores de umidade à base de massa ou de volume para cálculo de armazenagem, ou seja, considerar ou não a densidade do solo, não altera os pontos identificados como os mais representativos da área para o solo em questão. Esse fato é importante, pois indica que se pode selecionar o(s) ponto(s) pelas medidas gravimétricas.

Percebe-se, pelo quadro 5, que os valores de densidade do solo variam (diminuem) em profundidade, mas o coeficiente de variação por profundidade é bastante baixo, de 3,02 a 4,11\%, mostrando que a armazenagem volumétrica não difere da gravimétrica.

\section{Quadro 4. Parâmetros de regressão para armazenagem de água no solo $\left(S_{i j}\right)$}

\begin{tabular}{ccccccc}
\hline & \multicolumn{2}{c}{ Inclinação da regressão } & & \multicolumn{2}{c}{ Intercepto da regressão } \\
\cline { 2 - 6 } Periodo & Ano 1 & Ano 2 & Ano 3 & Ano 1 & Ano 2 \\
\cline { 3 - 6 } & 1 & 0,0387 & 0,0345 & 1 & 0,73 \\
Ano 1 & & 1 & $-0,016$ & 1,22 \\
Ano 2 & & 1 & 1 \\
\hline
\end{tabular}

Quadro 5. Resumo estatístico dos valores de densidade do solo $\left(\mathrm{kg} \mathrm{m}^{-3}\right)$, para cada profundidade de amostragem

Profundidade Média Mediana L. Mín. L. Máx. Amp. Total Desvio-padrão Curtose Assimetria CV

\begin{tabular}{|c|c|c|c|c|c|c|c|c|c|}
\hline $\mathrm{m}$ & & & & & & & & & $\%$ \\
\hline 0,30 & 1709 & 1704 & 1553 & 1842 & 288 & 70,26 & $-0,32$ & 0,25 & 4,11 \\
\hline 0,50 & 1659 & 1653 & 1532 & 1801 & 269 & 50,14 & 0,96 & 0,40 & 3,02 \\
\hline 0,70 & 1617 & 1621 & 1499 & 1758 & 259 & 58,66 & $-0,30$ & $-0,17$ & 3,63 \\
\hline 0,90 & 1583 & 1583 & 1464 & 1681 & 218 & 52,39 & $-0,19$ & 0,02 & 3,31 \\
\hline 1,10 & 1581 & 1574 & 1469 & 1852 & 383 & 63,69 & 7,33 & 0,33 & 4,03 \\
\hline
\end{tabular}




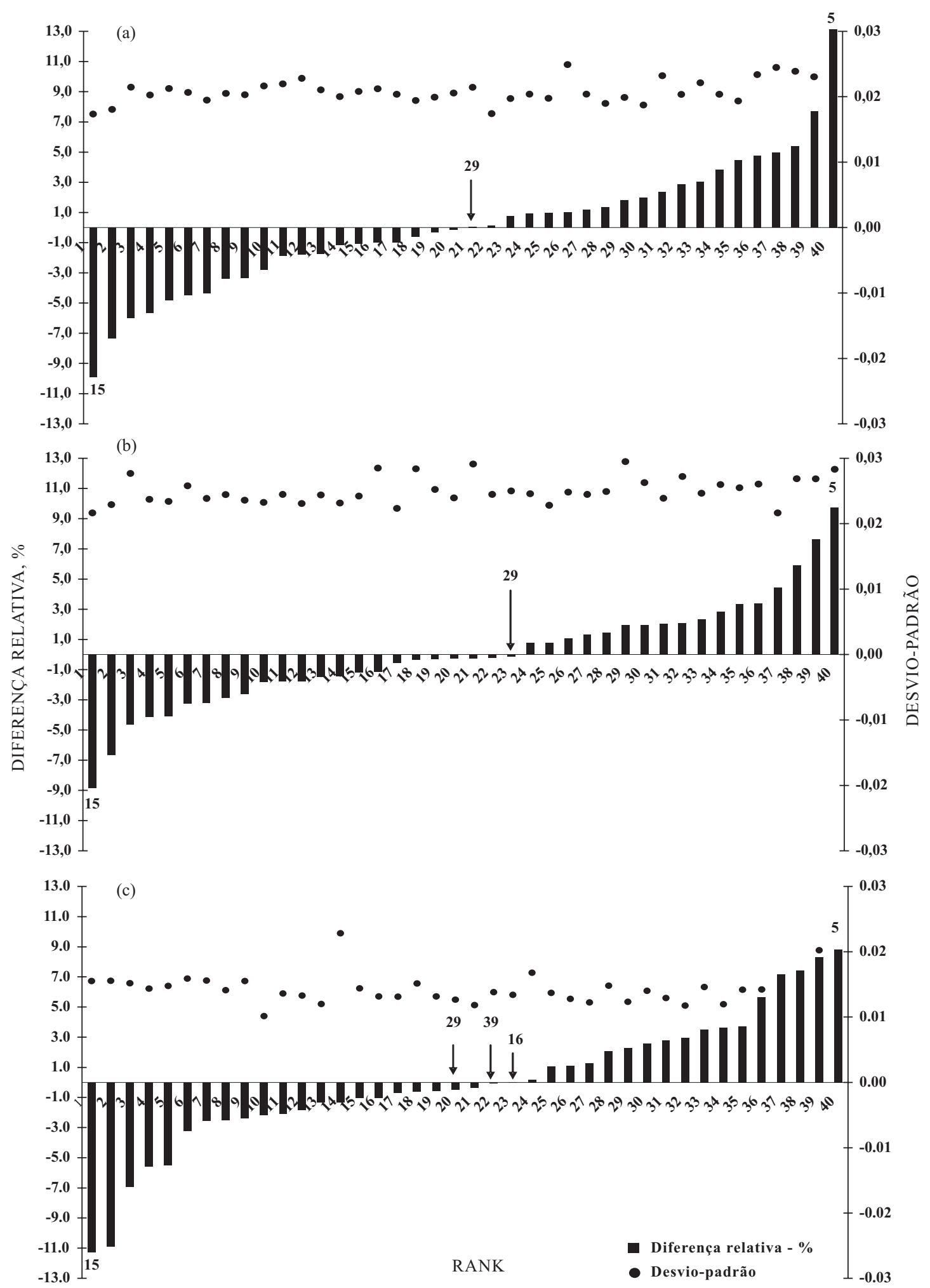

Figura 3. Diferença relativa média e respectivo desvio-padrão para o ano 1 (a), ano 2 (b) e ano 3 (c) com base na armazenagem de água no solo, listada do menor para o maior valor. Os números referem-se aos locais de medidas.

Melo Filho \& Libardi (2005), estudando a estabilidade temporal do teor de água no solo e do potencial mátrico da água na determinação da condutividade hidráulica em um perfil instantâneo, verificaram que os resultados pelo método da estabilidade temporal permitiram identificar, com 
exatidão, os locais mais adequados para as amostragens, possibilitando a redução do número de amostras necessárias e do custo de execução do esforço amostral para o planejamento de sistema de uso e manejo de água na agricultura.

Na figura 4 encontram-se a representação gráfica da média geral da armazenagem de água no solo e os pontos que mais se aproximaram da média, ou seja, os pontos que apresentaram estabilidade temporal pela técnica da estabilidade de Vachaud et al. (1985). Essa representação possibilita melhor visualizar o comportamento dos pontos em relação à média, confirmando a escolha deles, cuja diferença relativa foi mais próxima de zero e com menor desvio-padrão.

Esse método de escolha do ponto amostral tem aplicação bastante prática, visto que dá a indicação de como se pode realizar a amostragem com segurança da representatividade da área e reduzir o número delas, mesmo para propriedades de grande variabilidade, diminuindo o custo e o tempo despendido, podendo ser utilizada em áreas comerciais com finalidades diversas. Por exemplo, em áreas irrigadas poder-se-
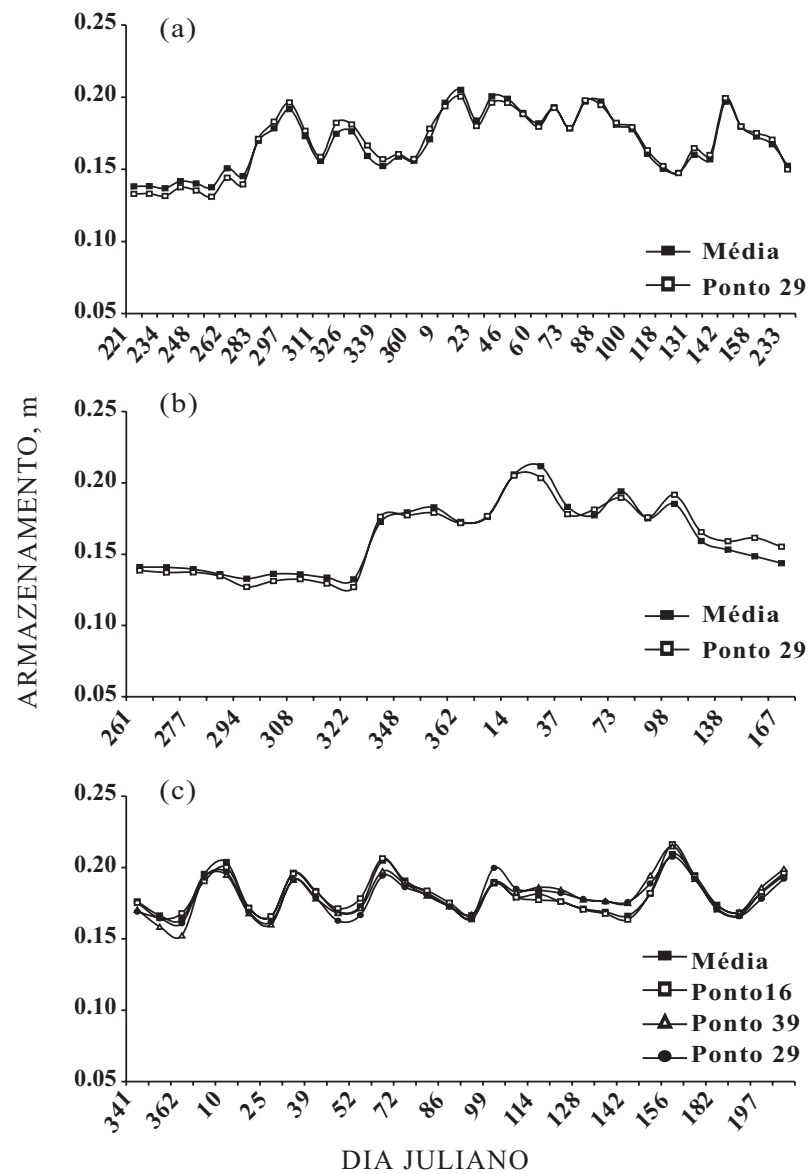

Figura 4. Comparação entre o armazenamento médio de água no solo e o armazenamento de água nas posições com estabilidade temporal para o ano 1 (a), ano 2 (b) e ano 3 (c). iam determinar pontos de amostragem que representam a média de toda a área e, neles, monitorar a disponibilidade de água por meio de tensiômetros. Dessa forma, há melhor controle do sistema, reduzindo custos e impactos ambientais gerados pelo uso desnecessário de água e eventual perda de solo devido à sua saturação excessiva.

Neste trabalho, qualquer um dos pontos escolhidos mostrou estabilidade temporal e poderia ser escolhido para representar a área. O ponto 29 foi o que mais se aproximou da média nos três anos de avaliação, para armazenagem volumétrica; portanto, poderia ser o ponto de amostragem representativo da área no monitoramento da armazenagem de água no solo para fins de estudo de balanço hídrico da cultura e outras aplicações. Para armazenagem gravimétrica, o ponto mais representativo foi o 28 (Rocha et al., 2005), distante apenas $4 \mathrm{~m}$ do ponto 29 .

\section{CONCLUSÕES}

1. Os valores de armazenagem da água no solo apresentaram-se estáveis ao longo do tempo e foram avaliados pelo coeficiente de correlação de Spearman e pela técnica da diferença relativa média, que permitiu também identificar pontos que representam a média da área, independentemente do tempo de amostragem. Por meio de semivariogramas, foi possível verificar a variabilidade espacial, com dependência de 17,10 m.

2. Como os valores se repetiram ao longo dos três anos com considerável margem de acerto, é possível estudos semelhantes com coletas de dados de um ano.

3 . Os valores de armazenagem de água no solo apresentaram dependência espacial de $17,10 \mathrm{~m}$ e estabilidade temporal. No caso desse solo, a armazenagem volumétrica não diferiu da armazenagem gravimétrica. Na avaliação da estabilidade temporal da armazenagem de água no solo, os métodos do coeficiente de posição de Spearman e da diferença relativa demonstraram a estabilidade dos valores ao longo dos três anos e permitiram identificar pontos que representam o valor médio da área. Como os valores se repetiram ao longo dos três anos com considerável margem de acerto, é possível realizar estudos semelhantes com coletas de dados de apenas um ano.

\section{LITERATURA CITADA}

ANDRIOTTI, J.L.S. Fundamentos de estatística e geoestatística. São Leopoldo, Unisinos, 2004. 165p.

ANGELOCCI, L.R. Água na planta e trocas gasosas/energéticas com a atmosfera: Introdução ao tratamento biofísico. Piracicaba, Edição do Autor, 2002. 272p. 
CAMBARDELLA, C.A.; MOORMAN, T.B.; NOVAK, J.M.; PARKIN, T.B.; KARLEN, D.L.; TURCO, R.F. \& KONOPKA, A.E. Field-scale variability of soil properties in Central Iowa soils. Soil Sci. Soc. Am. J., v.58, p.15011511,1994

EMPRESA BRASILEIRA DE PESQUISA AGROPECUÁRIA EMBRAPA. Centro Nacional de Pesquisa de Solos. Manual de métodos de análise de solo. 2 ed. Rio de Janeiro, 1997. 212p.

EMPRESA BRASILEIRA DE PESQUISA AGROPECUÁRIA EMBRAPA. Sistema brasileiro de classificação de solos. Brasília, 1999. 412p.

GONÇALVES, A.C.A.; FOLEGATTI, M.V. \& SILVA, A.P. Estabilidade temporal da distribuição espacial da umidade do solo em área irrigada por pivô central. R. Bras. Ci. Solo, 23:155-164, 1999.

GONCALVES, A.C.A.; FOLEGATTI, M.V. \& VIANA, J.D. Análises exploratória e geoestatística da variabilidade de propriedades físicas de um Argissolo Vermelho. Acta Sci., 23:1149-1157, 2001.

KACHANOSKI, R.G. \& De JONG, E. Scale dependence and the temporal persistence of spatial patterns of soil water storage. Water Res. Res, 24:85-91, 1988.

LIBARDI, L.P.; MANFRON, P.A.; MORAES, S.O. \& TUON, R.L. Variabilidade da umidade gravimétrica de um solo hidromórfico. R. Bras. Ci. Solo, 20:1-12, 1996.

LIBARDI, P.L. Dinâmica da água no solo. São Paulo, EDUSP, 2005. 335p.

MALLANTS, D.; MOHANTY, B.P.; JACQUES, D. \& FEYEN, J. Spatial variability of hydraulic properties in a multilayered soil profile. Soil Sci., 161:167-181, 1996.

MATHERON, G. The theory of regionalized variables and its application. Les Cahiers du Centre de Morphologie Mathematique, Fas. 5, C. G. Fontainebleau. 1971.
MELO FILHO, J.F. \& LIBARDI, P.L. Estabilidade temporal de medidas do teor e do potencial mátrico da água no solo em uma transeção. R. Bras. Ci. Solo, 29:497-506, 2005

MERCANTE, E.; URIBE-OPAZO, M.A. \& SOUZA, E.G. Variabilidade espacial e temporal da resistência mecânica do solo à penetração em áreas com e sem manejo químico localizado. R. Bras. Ci. Solo, 27:1149-1159, 2003.

RIBEIRO JUNIOR, P.J. \& DIGGLE, P.J. GeoR: A package for geostatistical analysis. R.News, 1:15-18, 2001.

ROCHA, G.C. Estabilidade temporal da distribuição espacial da armazenagem, do gradiente de potencial total e do potencial mátrico da água, em um solo cultivado com citros. Piracicaba, Escola Superior de Agricultura "Luiz de Queiroz", 2004. (Tese de Doutorado)

ROCHA, G.C.; LIBARDI, P.L.; CARVALHO, L.A. \& CRUZ, A.C.R. Estabilidade temporal da distribuição espacial da armazenagem de água em um solo cultivado com citros. R. Bras. Ci. Solo, 29:42-50, 2005.

RUIZ, P.M.E. \& UTSET, S.A. Uso del modelo swacrop en la estimatión de las necesidade hídricas de la papa (Solanum tuberosum L.), determinación de las propriedades hidráulicas del suelos. Revista Ciências Técnicas Agropecuárias, v.18, 1999. Disponível em:<http:// www.redepapa.org/propiedadsuelo.pdf $>$. Acesso em $06 \mathrm{de}$ set. 2005.

STATSOFT. Statistica Release 7.1. Tulsa, 2005.

VACHAUD, G.; PASSERAT, A.S.; BALABANIS, P. \& VAUCLIN, M. Temporal stability of spatially measured soil water probability density function. Soil Sci. Soc. Am. J, 49:822-827, 1985.

WARRICK, A.W. \& NIELSEN, D.R. Spatial variability of soil physical properties in the field. In: HILLEL, D. Applications of soil physics. New York, Academic Press, 1980. p.319344 . 